\title{
CAPÍTULO 03: UTILIZAÇÃO DE SISTEMAS DE LIBERAÇÃO DE FÁRMACOS TRANSDÉRMICOS NO TRATAMENTO DE PSORÍASE: UMA REVISÃO INTEGRATIVA
}

\author{
CAPÍTULO 03: USO DE SISTEMAS DE LIBERACIÓN TRANSDÉRMICA DE \\ DROGAS EN EL TRATAMIENTO DE LA PSORIASIS: UNA REVISIÓN \\ INTEGRATIVA
}

\section{CHAPTER 03: USE OF TRANSDERMAL DRUG DELIVERY SYSTEMS IN THE TREATMENT OF PSORIASIS: AN INTEGRATIVE REVIEW}

\author{
Wesley Ferreira de Moraes Brandão ${ }^{1}$; Thaíse Caroline da Silva Lima²; Luana Angélica Aires Rodrigues Jordão ${ }^{3}$; \\ Maria Eduarda Pires Lima ${ }^{4}$; Jeymesson Raphael Cardoso Vieira ${ }^{5}$
}

DOI: https://doi.org/10.31692/978-65-88970-04-1.31-44

\section{RESUMO}

A psoríase é uma doença crônica, não transmissível, dolorosa, incapacitante que possui caráter inflamatório e autoimune. Ela tem como principal característica clínica lesões cutâneas eritematosas e escamosas, geralmente localizadas nas articulações (cotovelos, joelhos) e couro cabeludo. $\mathrm{O}$ tratamento convencional da doença consiste em medicamentos que necessitam de alta dosagem e possuem efeitos colaterais que, quando administrados oralmente, podem acarretar malefícios ao trato digestivo, bem como quando por via tópica, possuem baixa permeabilidade e absorção cutânea. Uma nova abordagem de transporte de drogas por meio de um veículo, denominada de liberação de fármacos transdérmicos (SLFT), destaca-se por proporcionar maior eficiência de aprisionamento, produtividade, biocompatibilidade aprimorada e ação sustentada local de medicamentos no tratamento de psoríase, podendo auxiliar na redução da dose dos fármacos, assim como de efeitos adversos, reduzindo também a carga de tratamento dos pacientes. Este artigo tem como objetivo identificar a utilização de sistemas de liberação de fármacos transdérmicos no tratamento de pacientes acometidos por psoríase. Para isso, foi realizada uma pesquisa bibliográfica do tipo revisão integrativa. A busca ocorreu em outubro de 2020 nas seguintes bases de dados: EMBASE, MEDLINE, CENTRAL, SCOPUS, Web of Science e LILACS. A seleção e extração de dados dos artigos foram realizadas por dois pesquisadores independentes. Os estudos elegíveis para inclusão foram artigos originais com relatos do uso de um sistema de liberação de fármacos transdérmicos no tratamento de psoríase e que estivessem disponíveis na íntegra. Um instrumento de coleta de dados foi construído com indagações sobre a identificação do artigo, aspectos, bem como os desfechos dos artigos. Os resultados obtidos mostraram que os SLTF foram eficientes na entrega percutânea de drogas através da pele, além de apresentarem efeitos antiinflamatório significativos, reduzindo as citocinas pró-inflamatórias. Os dados demonstraram também o efeito oclusivo caracterizado pela formação de uma camada fina na pele. A análise da amostra permitiu identificar o uso de SLFT no tratamento de psoríase, no qual estes apresentaram baixa toxicidade e redução de efeitos adversos. Logo, conclui-se que, de acordo com os artigos da amostra, a utilização dơs SLTF mostrou-se eficaz no tratamento da doença, devido sua capacidade de entrega de drogas con potencial anti-inflamatório. Além disso, os artigos apontaram que os SLTF oferecem menos riscos ao paciente, menos efeitos colaterais, redução de dose e, consequentemente, carga do tratamento. Palavras-Chave: Tratamento, Psoríase, Sistemas de Liberação de Fármacos Transdérmicos.

\section{RESUMEN}

\footnotetext{
${ }^{1}$ Enfermagem, Universidade Federal da Paraíba, owesleybrandao@gmail.com

${ }^{2}$ Farmácia, Universidade Federal da Paraíba, thaise.carolinesl@gmail.com

${ }^{3}$ Odontologia, Universidade Federal da Paraíba, luanaairesjordao@gmail.com

${ }^{4}$ Enfermagem, Universidade Federal da Paraíba, eduardapires.ufpb@gmail.com

${ }^{5} \mathrm{PhD}$, Universidade Federal de Pernambuco, jeymesson@gmail.com
} 
La psoriasis es una enfermedad crónica, no transmisible, dolorosa e invalidante que tiene un carácter inflamatorio y autoinmune. Su principal característica clínica son lesiones cutáneas eritematosas y escamosas, generalmente localizadas en las articulaciones y cuero cabelludo. Su tratamiento convencional enfermedad consiste en fármacos que requieren altas dosis y tienen efectos secundarios que, administrados por vía oral, pueden causar daño al tracto digestivo, así como por vía tópica, tienen baja permeabilidad y absorción cutánea. Un nuevo enfoque para el transporte de fármacos por vehículo, llamado liberación transdérmica de fármacos (LTF), se destaca proporcionando una mayor eficiencia de captura, productividad, biocompatibilidad mejorada y acción local sostenida de los fármacos en el tratamiento de la psoriasis, lo que puede ayudar en la reducción de la dosis de fármacos, así como de los efectos adversos, reduciendo también la carga de tratamiento de los pacientes. Este artículo tiene como objetivo identificar el uso de sistemas de administración de fármacos transdérmicos en el tratamiento de pacientes con psoriasis. Para ello se realizó una investigación bibliográfica tipo revisión integradora. La búsqueda se realizó en octubre de 2020 en las siguientes bases de datos: EMBASE, MEDLINE, CENTRAL, SCOPUS, Web of Science y LILACS. La selección y extracción de datos de los artículos fue realizada por dos investigadores independientes. Los estudios elegibles para su inclusión eran artículos originales con informes sobre el uso de un sistema de administración de fármacos transdérmicos en el tratamiento de la psoriasis y que estaban disponibles en su totalidad. Se construyó un instrumento de recolección de datos con preguntas sobre la identificación del artículo, aspectos, así como los resultados de los artículos. Los resultados obtenidos mostraron que los LTF eran eficientes en el suministro percutáneo de fármacos a través de la piel, además de tener importantes efectos antiinflamatorios, reduciendo las citocinas proinflamatorias. Los datos también demostraron el efecto oclusivo caracterizado por la formación de una fina capa sobre la piel. El análisis de la muestra permitió identificar el uso de LTF en el tratamiento de la psoriasis, en la que presentaban baja toxicidad y reducidos efectos adversos. Por tanto, se concluye, según los artículos de la muestra, el uso de LTF demostró-se eficaz en el tratamiento de la enfermedad, debido a su capacidad para administrar fármacos con potencial antiinflamatorio. Además, los artículos señalaron que los LTF ofrecen menos riesgo para el paciente, menos efectos secundarios, reducción de dosis y, en consecuencia, carga de tratamiento.

Palabras clave: Tratamiento, Psoriasis, Sistemas de administración transdérmica de fármacos.

\begin{abstract}
Psoriasis is a chronic, non-transmissible, painful, disabling disease that has an inflammatory and autoimmune character. Its main clinical feature is erythematous and scaly skin lesions, usually located in the joints (elbows, knees) and scalp. Conventional treatment of the disease consists of drugs that require high dosage and have side effects that, when administered orally, can cause harm to the digestive tract, as well as when topically, have low permeability and cutaneous absorption. A new approach to transporting drugs by vehicle, called transdermal drug delivery system (TDDS), stands out for providing greater trapping efficiency, productivity, improved biocompatibility and sustained local action of drugs in the treatment of psoriasis, which can help in reducing the dose of drugs, as well as adverse effects, also reducing the treatment burden of patients. This article aims to identify the use of transdermal drug delivery systems in the treatment of patients with psoriasis. For that, an integrative review type bibliographic research was carried out. The search took place in October 2020 in the following databases: (EMBASE), MEDLINE, CENTRAL, SCOPUS, Web of Science and LILACS. The selection and data extraction of the articles were carried out by two independent researchers. The studies eligible for inclusion were original articles with reports of the use of a transdermal drug delivery system in treatment of psoriasis and that were available in full. A data collection instrument was built questions about the identification of the article, aspects, as well as the outcomes of the articles. results obtained showed that TDDSs were efficient in the percutaneous delivery of drugs throug skin, in addition to having significant anti-inflammatory effects, reducing pro-inflammatory cyt The data also demonstrated the occlusive effect characterized by the formation of a thin skin. The analysis of the sample made it possible to identify the use of TDDS in th psoriasis, in which they presented low toxicity and reduced adverse effects. Therefore, that, according to the sample articles, the use of TDDS proved to be effective in disease, due to its ability to deliver drugs with anti-inflammatory potential. I pointed out that TDDSs offer less risk to the patient, fewer side effects, consequently, treatment burden.
\end{abstract}


Keywords: Treatment. Psoriasis. Transdermal Drug Delivery Systems.

\section{INTRODUÇÃO}

Segundo a organização mundial da saúde (OMS), a psoríase é uma doença crônica, de caráter não transmissível, dolorosa, desfigurante e incapacitante para qual não há cura, possuindo grande impacto negativo sobre a qualidade de vida dos pacientes (OMS, 2016). Esta doença atinge 2-5\% da população mundial (SALA; ELAISSARI; FESSI, 2016), sendo distribuída de maneira desigual ao redor do mundo, com maior frequência em países de alta renda e regiões com populações mais velhas (PARISI et al., 2020). De acordo com a Sociedade Brasileira de Dermatologia (2016), a prevalência da psoríase no país varia entre 1,10 e 1,50\%, apresentando grande variabilidade nas regiões Norte $(0,92 \%)$ e Sudeste $(1,88 \%)$.

A psoríase possui caráter inflamatório e autoimune, tendo como principal característica clínica lesões cutâneas eritematosas e escamosas geralmente localizadas nas articulações (cotovelos, joelhos) e couro cabeludo, no entanto ressalta-se que qualquer região do corpo pode ser acometida (SALA; ELAISSARI; FESSI, 2016). Estas lesões são induzidas por respostas imunológicas, que provocam proliferação e diferenciação prejudicada de queratinócitos, resultando em acantose epidérmica, hiperqueratose e paraqueratose (BOEHNCKE; BREMBILLA, 2018).

O tratamento da psoríase varia de acordo com sua severidade, em que tem-se como finalidade primária a atenuação das alterações morfológicas da pele através da redução das respostas imunes causadas pela progressão da doença (SALA; ELAISSARI; FESSI, 2016). O tratamento medicamentoso convencional oferece como desvantagens altas doses e efeitos colaterais relacionados ao trato digestivo, quando administrados oralmente, bem como baixa permeabilidade e absorção cutânea, quando administrados por via tópica (RAPALLI et al., 2020).

Uma nova abordagem terapêutica de transporte de drogas por meio de um veículo, denominada sistema de liberação de fármacos transdérmicos (SLFT), destaca-se por proporcionar maior eficiência de aprisionamento, produtividade, biocompatibilidade aprimorada e ação sustentada local de medicamentos no tratamento de psoríase (KUMAR et al., 2020). O desenvolvimento de métodos avançados de SLFT pode auxiliar na redução da dose e frequência de fármacos, assim como de efeitos adversos, reduzindo também a carga de tratamento dos pacientes (RAPALLI et al., 2020).

Diante do constante avanço no campo dos SLFT, e considerando a escassez de artigos 
de revisão sobre a temática, especialmente em português, evidencia-se a relevância deste estudo ao realizar um apanhado geral do uso de SLFT para o tratamento de psoríase. Desse modo, garantindo a profissionais e pesquisadores fácil acesso informações acerca deste tópico e incitando reflexões para realizações de pesquisas futuras na área.

Para tal, realizou-se uma revisão integrativa com a finalidade de reunir e sintetizar evidências atuais sobre a temática para responder a seguinte pergunta norteadora da pesquisa: quais são os sistemas de liberação transdérmicas de fármacos já utilizados no tratamento de psoríase? Sendo assim, o estudo tem como objetivo identificar a utilização de sistemas de liberação de fármacos transdérmicos no tratamento de pacientes acometidos por psoríase.

\section{FUNDAMENTAÇÃO TEÓRICA}

A pele constituída por três camadas: epiderme, derme e hipoderme. A epiderme possui origem embriológica da ectoderme e é formada por um epitélio estratificado pavimentoso queratinizado. A camada subsequente à epiderme é a derme, derivada da mesoderme, a qual é composta por tecido conjuntivo frouxo (derme papilar), que conta com células, vasos sanguíneos, fibras, substância fundamental amorfa, além do tecido conjuntivo denso não modelado (derme reticular), rico em fibras colágenas e elásticas. A última camada, a hipoderme, une a pele aos órgãos subjacentes (fáscia subcutânea) e é composta por tecido adiposo e por conjuntivo frouxo (JUNQUEIRA et al., 2017).

A epiderme é constituída por cinco camadas distintas: estrato basal, estrato espinhoso, estrato granuloso, estrato lúcido e estrato córneo. Ressalta-se ainda a existência do estrato lúcido, o qual é considerado uma subdivisão do estrato córneo visível apenas na pele espessa. (PAWLINA; ROSS, 2018). Os queratinócitos presentes no estrato granuloso modificam sua expressão gênica e sintetizam citoqueratinas, involucrina, loricrina e filagrina, dando origem aos grânulos de querato-hialina. Além disso, ainda é sintetizado colesterol, ácidos graxos livre esfingolipídios, ceramidas e glicolipídios acondicionados em corpos lamelares. A partir dessa área se forma o estrato córneo, onde os queratinócitos formam muitas fibrilas de queratina (queratina mole) e compactam esse conteúdo (JUNQUEIRA et al., 2017).

O estrato córneo é formado por camadas sequenciais de células hexagonais achatadas de queratina denominadas corneócitos. Segundo o modelo de "bricks and corneócito corresponderia a um tijolo (brick), com permeabilidade quase inexistente, que estaria ligado a outro tijolo adjacente por intermédio de uma argamassa (mortar), que corresponderia a uma lacuna lipídica onde ocorre a difusão, sendo considerado o principal canal 
de permeação de xenobióticos (SEBASTIA-SAEZ et al., 2020).

Estas camadas da epiderme, especialmente o estrato córneo, formam uma barreira impermeável à água e podem dificultar a absorção tópica de moléculas. Nesse contexto, os sistemas de liberação de fármacos transdérmicos (SLFT) buscam vencer a barreira criada pela pele e garantir a distribuição de fármacos localmente e sistemicamente (RENADE, 1991). Como a psoríase é patologia de interesse do estudo, destaca-se ainda que alterações histológicas características da doença, como redução da membrana basal, localizada na junção dermoepidérmica, pode favorecer a penetração de substâncias através da pele (GORZELANNY et al., 2020).

Os SLFT são considerados uma técnica de administração parenteral de medicamentos não invasiva, que pode ser aplicada e removida facilmente e que, diferentemente da administração por via oral, evita o metabolismo de primeira passagem no fígado (QI, 2020). Quanto às classificações dos SLFT, tem-se que estes podem ser de: primeira geração, que compreendem drogas de baixo peso molecular/potência; segunda geração, que compreendem os sistemas com intensificadores de penetração e terceira geração, que correspondem aos sistemas com técnicas físicas para vencer as propriedades de barreira da pele (NEUPANE et al., 2020).

Uma série de elementos podem influenciar a performance do transporte de fármacos através da pele, e por este motivo, devem ser levados em consideração no desenvolvimento de um SLFT, tais como: a estrutura da pele e suas propriedades; a molécula penetrante e sua relação físico-química com a pele e plataforma de liberação; a plataforma ou o sistema de liberação transportando a molécula penetrante e, por fim, consonância entre a pele, o penetrante e o sistema de liberação como um todo (RENADE, 1991).

De maneira geral, tem-se que os fármacos penetram na pele através de três rotas: a transcelular, que ocorre por difusão através dos corneócitos, na qual o fármaco entra em contato com as regiões de baixo teor de lipídios no citoplasma dos corneócitos; a intracelular, que ocorre por difusão através da matriz de lipídios, em que as moléculas hidrofílicas utilizam a porção polar das lamelas de lipídio, enquanto que as moléculas lipofílicas fazem uso da cauda lipídica e, por fim, a rota apendágica, que ocorre por difusão de moléculas através de glândulas sudoríparas, sebáceas e folículos capilares, sendo considerada uma rota secundária de permeação transdérmica pois estes apêndices compõem cerca de $0.1 \%$ da pele (NEUPANE et al., 2020).

No contexto do tratamento de psoríase, os SLFT oferecem a possibilidade de: fluidificar o estrato córneo como um componente de estabilidade hidrofílica-hidrofóbica; fazer uso da 
nanotecnologia para apresentar tamanhos e formas que promovem o contato próximo da superfície da pele, e consequentemente, melhoram a penetração do fármaco; permitir que o fármaco permaneça na superfície da da pele para uma liberação controlada; e apresentar propriedades oclusivas que contribuem para a diminuição de perda de água transepidérmica (KUMAR et al., 2020).

\section{METODOLOGIA}

Trata-se de uma pesquisa bibliográfica do tipo revisão integrativa que tem como finalidade realizar uma síntese de evidências atuais de uma área de conhecimento, construindo uma conclusão integrada e unificada para responder um problema particular de pesquisa (WHITTEMORE; KNALF et al., 2005). A pesquisa se desenvolveu em seis etapas, sendo elas: elaboração da pergunta norteadora, busca nas bases de dados, coleta de dados, análise dos artigos incluídos na amostra, discussão dos resultados e apresentação da revisão (SOUZA; CARVALHO, 2010).

A pergunta norteadora foi elaborada com a estratégia PICO, acrônimo para "População, Intervenção, Comparação e Desfecho (do inglês, Outcome) (METHLEY et al., 2014). Desse modo, P - correspondeu a psoríase; I - sistema de liberação de fármacos transdérmico; C - não utilizado no estudo; $\mathrm{O}$ - tratamento da psoríase.

A busca ocorreu em outubro de 2020 nas seguintes bases de dado: Excerpta Medica (EMBASE), Medical Literature Analysis and Retrieval System Online (MEDLINE) via PubMed, Cochrane Central Register of Controlled Trials (CENTRAL), SCOPUS, Web of Science e Literatura Latino-Americana e do Caribe em Ciências da Saúde (LILACS). Inicialmente foram verificadas nas seis bases a existência de descritores ou termos controlados. Foram realizadas combinações com o operador booleano “AND” e Descritores em Saúde (DECS), termos Medical Subject Heading (MESH) e termos Emtree tais como: Administração Cutânea; Sistema de Liberação de Medicamentos; Psoríase; Drug; Administration, Cutaneous Drug delivery systems e Psoriasis. A tabela 1 apresenta as combinações utilizadas nas diferentes bases de dados.

A seleção e extração de dados dos artigos foram realizadas por dois pesquisadores independentes. Os estudos elegíveis para inclusão foram artigos originais com relatos do uso de um sistema de liberação de fármacos transdérmicos no tratamento estivessem disponíveis na íntegra via servidor proxy da Universidade Federal da Paraíba. Os critérios de exclusão foram artigos que não utilizassem modelos ou amostras de psoríase no 
desenvolvimento do seu estudo.

Tabela 1: Estratégia de busca utilizada.

\begin{tabular}{ccc}
\hline Base de dado & Estratégia de busca & Resultado \\
\hline $\begin{array}{c}\text { MEDLINE/ } \\
\text { PUBMED }\end{array}$ & $\begin{array}{c}\text { (("Administration, Cutaneous"[Mesh]) AND "Drug Delivery } \\
\text { Systems"[Mesh]) AND "Psoriasis"[Mesh] }\end{array}$ & 8 \\
\hline & $\begin{array}{c}\text { Administration, Cutaneous in Title Abstract Keyword AND } \\
\text { Drug Delivery Systems in Title Abstract Keyword AND } \\
\text { Psoriasis in Title Abstract Keyword - (Word variations have } \\
\text { been searched) }\end{array}$ & 5 \\
\hline EMBASE & $\begin{array}{c}\text { cutaneous drug administration' AND 'drug delivery system' AND } \\
\text { psoriasis }\end{array}$ & 3 \\
& $\begin{array}{c}\text { TITLE-ABS-KEY ( '"cutaneous AND drug AND administration' } \\
\text { AND 'drug AND delivery AND system' AND psoriasis ) AND ( } \\
\text { LIMIT-TO ( ACCESSTYPE(OA) ) ) AND ( LIMIT-TO ( } \\
\text { DOCTYPE , "ar" ) ) }\end{array}$ & 8 \\
\hline WEB OF & $\begin{array}{c}\text { (TS=("'cutaneous AND drug AND administration' AND 'drug } \\
\text { AND delivery AND system' AND psoriasis) ) AND TIPOS DE } \\
\text { DOCUMENTO: (Article) }\end{array}$ & 2 \\
\hline LILACS & $\begin{array}{c}\text { Administração Cutânea [Palavras] and Sistemas de Liberação de } \\
\text { Medicamentos [Palavras] and Psoríase [Palavras] }\end{array}$ & 0 \\
\hline
\end{tabular}

Fonte: Elaborada pelos autores (2020).

Um instrumento de coleta de dados foi construído pelos pesquisadores com indagações sobre a identificação do artigo (título, autores, idioma, ano de publicação e periódico), aspectos metodológicos (amostra, fármaco, modelo de SLTF, geração do STLF), bem como os desfechos dos artigos.

Os dados extraídos foram dispostos em uma planilha de excel para que fossem analisados pela Análise de Conteúdo. Esta técnica de análise conta com: pré-análise, em que todo material selecionado para análise foi lido exaustivamente; exploração do material, que tratou de codificar e agrupar as informações da amostra final em categorias a fim de facilitar a análise; e interpretação, com o tratamento dos resultados e análise comparativa (BARDIN, 2011).

\section{RESULTADOS E DISCUSSÃO}

A busca nas bases de dados resgatou 26 produções, sendo oito na MEDLINE (PubMed), três na EMBASE, cinco na CENTRAL, oito na SCOPUS, duas na Web of Science e zero na LILACS. Foram excluídos 14 artigos após a aplicação dos critérios de inclusão e exclusão, em 
seguida mais três artigos foram excluídos por não terem sido desenvolvidos com amostras ou modelos de psoríase (Figura 1).

Figura 1: Fluxograma de seleção de artigos.

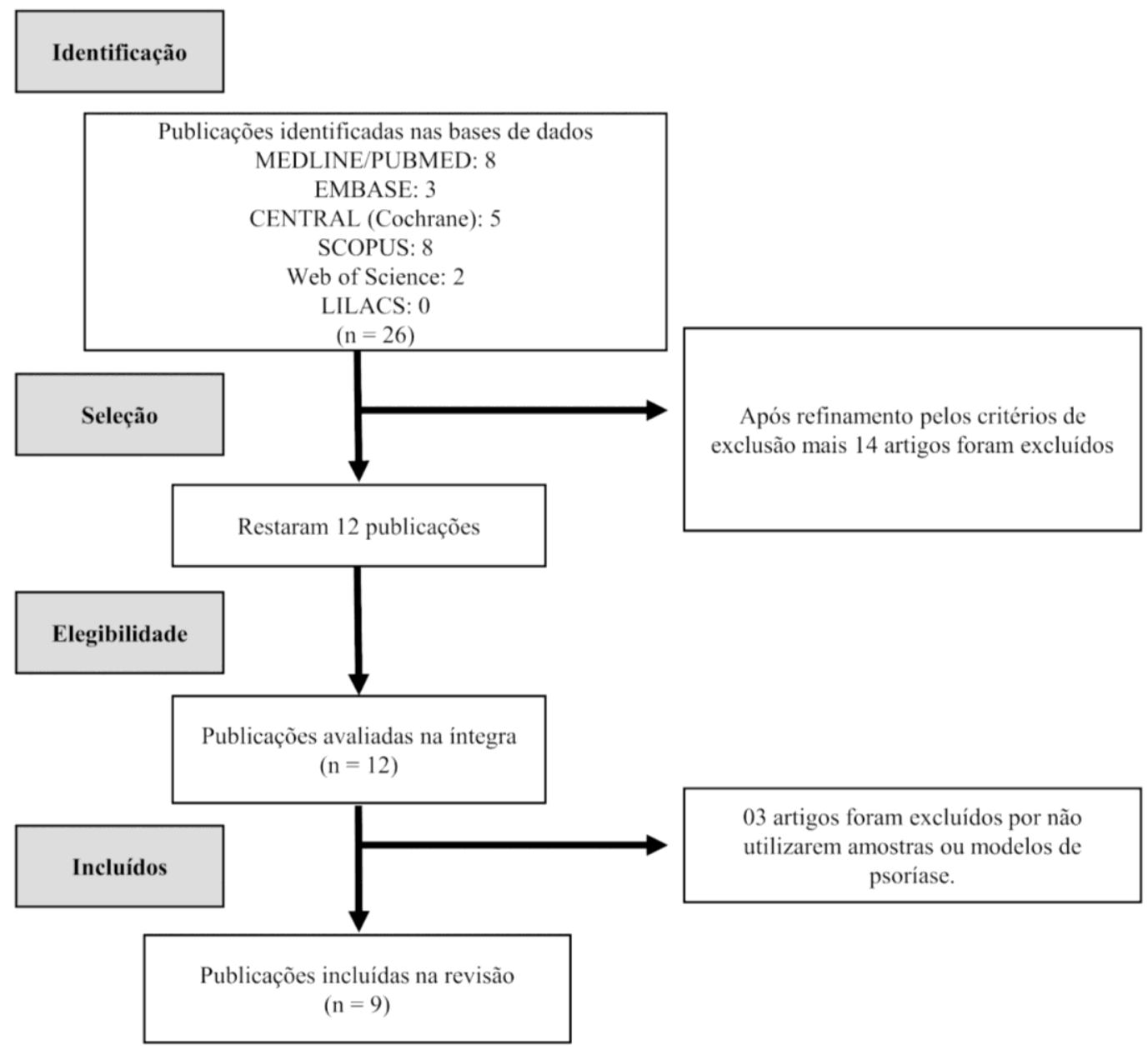

Fonte: Elaborada pelos autores (2020).

Desse modo, a amostra final foi composta por 9 artigos, os quais estão caracterizados segundo as variáveis de interesse no quadro 1 . Todos os artigos da amostra estavam no idioma inglês. Dentre os países onde as pesquisas foram realizadas, houve predomínio dos Estados Unidos da América (BOAKY et al., 2017; SOMAGONI et al., 2014; SHAH et al., 2012), seguidos da Índia (AGRAWAL et al., 2020; WADHWA et al., 2016), Reino Unido (DUNCAN et al., 1993), Coreia (KIM et al., 2018), Itália (SAPINO et al., 2017) e Brasil (DEPIERI et al., 2016). Quanto aos anos de publicação dos artigos, observou-se que estes (DUNCAN et al., 1993), 2012 (SHAH et al., 2012), 2014 (SOMAGONI et al., 2014), 2016 (WADHWA et al., 2016), 2017 (BOAKY et al., 2017; SAPINO et al., 2017), 2018 (KIM et al., 2018) e 2020 (AGRAWAL et al., 2020). 
Houve predominância dos pesquisas que realizaram tanto experimentos in vitro, geralmente com análises da liberação da droga, quanto in vivo, no qual em geral utilizou-se camundongos de laboratório (SHAH et al., 2012; AGRAWAL et al., 2020; KIM et al., 2018; BOAKY et al., 2017; WADHWA et al., 2016; SOMAGONI et al., 2014). Ademais, duas pesquisas realizaram somente experimentos in vitro (SAPINO et al., 2017; DEPIERI et al., 2016) ou apenas in vivo (DUNCAN et al., 1993). Além disso, observou-se que as células de difusão de Franz foram amplamente utilizadas pelos estudos da amostra para avaliação in vitro do SLTF (SHAH et al., 2012; BOAKY et al., 2017; WADHWA et al., 2016; SOMAGONI et al., 2014; SAPINO et al., 2017; DEPIERI et al., 2016).

No que tange os SLFT, identificou-se predominância de sistemas de terceira geração (BOAKY et al., 2017; WADHWA et al., 2016; SOMAGONI et al., 2014; SAPINO et al., 2017), que dispuseram de técnicas físicas para superar a barreira física da pele (NEUPANE et al., 2020). Ressalta-se ainda que alguns sistemas utilizados nos estudos da amostra (SAPINO et al., 2017 DEPIERI et al., 2016; KIM et al., 2018) apresentaram características de terceira e segunda geração, em que foram utilizados intensificadores de penetração (NEUPANE et al., 2020). Por fim, um estudo apresentou um sistema com características de primeira geração (DUNCAN et al., 1993) e um de segunda geração (SHAH et al., 2012).

Os dados mostraram que os SLTF foram eficientes na entrega percutânea de drogas até camadas profundas da pele (Shah et al., 2012; Agrawal et al., 2020; Kim et al., 2018; Boaky et al., 2017; Somagoni et al., 2014; Depieri et al., 2016). Sabe-se que diante das dificuldades do meio externo, como agentes químicos, físicos, biológicos, e a radiação, a pele exerce o papel de proteção ao nosso organismo. Assim, quando ela se encontra intacta, assume a posição de barreira contra a penetração e permeação de substâncias. Dessa maneira, esse fator pode ser limitante, uma vez que pode impedir ou dificultar a absorção do fármaco pela circulação sistêmica, comprometendo a permeação cutânea, ao passo que, também interfere na penetração cutânea, embora o fármaco não atravesse toda a epiderme (BABY et al., 2008).

Segundo Gomes e Gabriel (2006) , a maneira em que a pele permite que uma molécula a ultrapasse, podendo chegar à corrente sanguínea, é conhecida como permeabilidade cutânea. a qual depende de fatores como: tamanho da molécula e características físico-gu (AGOSTINI; SILVA, 2020). Um exemplo de medicamento que atua através da permeabilidade cutânea, são os adesivos transdérmicos de nicotina; além do componente fenol, usado na prática de peeling profundo, sozinho ou em conjunto com outros componentes, atuando tanto na penetração e permeação cutâneas (VELASCO et al

Ressalta-se ainda que a inflamação na pele, comumente encontrada na psoríase, 
ocasiona alterações histopatológicas, como a diminuição da membrana basal, o que atenua a troca de substâncias entre a epiderme e a derme (GORZELANNY et al., 2020). Nesse contexto, as lesões na pele ocasionadas por uma dermatite, por exemplo, tornam a penetração pelas camadas da pele mais relevantes, aumentando o número de partículas que atravessam a pele (SAPINO et al., 2017).

Quadro 1: Caracterização dos artigos da amostra final segundo as variáveis de interesse.

\begin{tabular}{|c|c|c|c|}
\hline Citação & Modelo & Fármaco & Principal desfecho \\
\hline $\begin{array}{l}\text { AGRAWAL } \\
\text { et al., } 2020\end{array}$ & $\begin{array}{l}\text { Carreadores de lipídios } \\
\text { nanoestruturados } \\
\text { carregados com } \\
\text { metotrexato }\end{array}$ & Metotrexato & $\begin{array}{l}\text { Liberação sustentada do fármaco por } 30 \\
\text { horas. Além de melhorar a resposta } \\
\text { terapêutica, o sistema causou uma redução } \\
\text { nos efeitos adversos, promovendo uma } \\
\text { melhor adesão do paciente. }\end{array}$ \\
\hline $\begin{array}{l}\text { BOAKY et } \\
\text { al., } 2017\end{array}$ & $\begin{array}{c}\text { Administração } \\
\text { transdérmica simultânea } \\
\text { de erlotinibe e siRNA de } \\
\text { IL36 } \alpha \text { através de } \\
\text { nanocarreadores de } \\
\text { lipídios (CYnLIP) }\end{array}$ & $\begin{array}{l}\text { Erlotinibe e } \\
\text { siRNA de } \\
\text { IL36 } \alpha\end{array}$ & $\begin{array}{l}\text { O aumento da entrega co-transdérmica de } \\
\text { erlotinibe e IL36 } \alpha \text { siRNA por placas } \\
\text { semelhantes a psoríase tratados eficazmente } \\
\text { CYnLIP em camundongos C57BL / } 6 \\
\text { (pontuação PASI de 1). Redução na } \\
\text { hiperplasia epidérmica (Ki67) e na } \\
\text { infiltração dérmica de citocinas } \\
\text { inflamatórias (IL36 } \alpha \text {, pSTAT3, TNF } \alpha \text {, } \\
\text { NFאB, IL23 e IL17). }\end{array}$ \\
\hline $\begin{array}{l}\text { DEPIERI et } \\
\text { al., } 2016\end{array}$ & $\begin{array}{l}\text { Uma nanodispersão de } \\
\text { siRNA baseada em fase } \\
\text { líquido-cristalina e } \\
\text { composta por } \\
\text { monooleína, ácido } \\
\text { oleico e polietilenimina }\end{array}$ & siRNA & $\begin{array}{l}\text { Eficácia na complexação do siRNA, com } \\
\text { alta taxa de captação celular ( } ~ 90 \%), \text { e } \\
\text { penetração cutânea do siRNA in vitro, sem } \\
\text { causar irritação na pele, resultando em uma } \\
\text { viabilidade } 4 \text { vezes maior da epiderme } \\
\text { humana reconstruída e uma liberação } 15,6 \\
\text { vezes menor de IL-1 } \alpha \text {. Em um único } \\
\text { tratamento por } 6 \text { h foi capaz de reduzir os } \\
\text { níveis de IL-6 extracelular em } 3,3 \text { vezes em } \\
\text { comparação com o tratamento de controle } \\
\text { em modelo de pele com psoríase. }\end{array}$ \\
\hline $\begin{array}{l}\text { DUNCAN et } \\
\text { al., } 1993\end{array}$ & $\begin{array}{l}\text { Ciclosporina A tópica } \\
\text { em uma formulação com } \\
\text { os intensificadores de } \\
\text { penetração } \\
\text { Propilenoglicol }(18 \%) \text { e } \\
\text { Azona }(2 \%)\end{array}$ & $\begin{array}{l}\text { Ciclosporina } \\
\text { A (Tópica) }\end{array}$ & $\begin{array}{l}\text { Melhora significativa nas placas de psoríase } \\
\text { dos pacientes tratados com a formulação. }\end{array}$ \\
\hline $\begin{array}{l}\text { KIM et al., } \\
\quad 2018\end{array}$ & $\begin{array}{l}\text { Sistema transdérmico } \\
\text { assistido por } \\
\text { nanopartículas lipídicas } \\
\text { em forma discóide } \\
\text { (DLNP) para } \\
\text { distribuição de } \\
\text { APTstat3-9R }\end{array}$ & APTstat3-9R & $\begin{array}{l}\text { Penetração na pele eficiente com um efeito } \\
\text { antiinflamatório em um modelo pré-clínico } \\
\text { de psoríase por meio da modulação da } \\
\text { sinalização de citocinas. }\end{array}$ \\
\hline
\end{tabular}




\begin{tabular}{|c|c|c|c|}
\hline $\begin{array}{l}\text { SAPINO et } \\
\text { al., } 2017\end{array}$ & $\begin{array}{l}\text { Preparações à base de } \\
\text { Metotrexato } \\
\text { transportadas por } \\
\text { Nanopartículas } \\
\text { semelhantes a MCM-41 }\end{array}$ & Metotrexato & $\begin{array}{c}\text { Presença confirmada das nanopartículas na } \\
\text { epiderme. Absorção pela pele favorecida } \\
\text { pela presença de manteiga de karité nas } \\
\text { formulações dérmicas. }\end{array}$ \\
\hline $\begin{array}{c}\text { SHAH et al., } \\
2012\end{array}$ & $\begin{array}{c}\text { Sistema de nanogel que } \\
\text { permeia a pele contendo } \\
\text { nanopartículas } \\
\text { poliméricas de duas } \\
\text { camadas modificadas na } \\
\text { superfície e um agente } \\
\text { gelificante. }\end{array}$ & $\begin{array}{l}\text { Spantide II e } \\
\text { Cetoprofeno }\end{array}$ & $\begin{array}{l}\text { O sistema tem potencial significativo para a } \\
\text { entrega percutânea de Spantide II e } \\
\text { Cetoprofeno às camadas mais profundas da } \\
\text { pele, com efeitos oclusivos. }\end{array}$ \\
\hline $\begin{array}{l}\text { SOMAGONI } \\
\text { et al., } 2014\end{array}$ & \begin{tabular}{|c} 
Nanomiemgel de \\
nanomicela e \\
nanoemulsão contendo \\
aceclofenaco e \\
capsaicina \\
\end{tabular} & $\begin{array}{c}\text { Aceclofenaco } \\
\text { e Capsaicina }\end{array}$ & $\begin{array}{c}\text { Aumento a permeação cutânea de } \\
\text { aceclofenaco e capsaicina, melhorando o } \\
\text { tempo de contato com a pele, hidratando-a e } \\
\text { formando uma camada fina na superfície da } \\
\text { pele. }\end{array}$ \\
\hline $\begin{array}{l}\text { WADHWA } \\
\text { et al., } 2016\end{array}$ & $\begin{array}{l}\text { Formulação de ácido } \\
\text { fusídico lipossomal } \\
\text { incorporado à um } \\
\text { sistema nano-portador }\end{array}$ & $\begin{array}{c}\text { Ácido fusídico } \\
\text { lipossomal }\end{array}$ & $\begin{array}{l}\text { Aumento no potencial de eficácia, segurança } \\
\text { e estabilidade do ácido fusídico com a } \\
\text { formulação lipossomal. }\end{array}$ \\
\hline
\end{tabular}

Fonte: Elaborado pelos autores (2020).

Além disso, após a análise dos dados, observou-se que os SLTF demonstraram exercer efeito anti-inflamatório significativos (SHAH et al., 2012; AGRAWAL et al., 2020; KIM et al., 2018; BOAKY et al., 2017), com redução de citocinas pró-inflamatórias (IL36 $\alpha$, pSTAT3, TNFa, NFкB, IL23 e IL17) (BOAKY et al., 2017). A produção de citocinas está intimamente ligada à fisiopatologia da psoríase, tendo em vista que esta doença se desenvolve em um ambiente inflamatório promovido por células do sistema imunológico que invadem a derme, como células T CD4+, células dendríticas e células de langerhans (SALA et al., 2016).

Nesse contexto, ratifica-se a importância da utilização de drogas com propriedades antiinflamatórias no desenvolvimento de SLTF para o tratamento de psoríase, desta forma, aliviando sintomas clássicos da doença como a vermelhidão, calor, espessura da pele, descamação, dor e inchaço (SOMAGONI et al., 2014). Portanto, reduzindo os impactos negativos que acompanham os pacientes afetados pela psoríase (OMS, 2016).

Outro efeito apontado pelos artigos da amostra final foi o efeito oclusivo caracterizado pela formação de uma camada fina na pele (SHAH et al., 2012; SOMAGONI et al., 2014). Como consequência deste evento, as células que compõe o estrato córneo evitam a perda de água transepidérmica, acarretando hidratação desta camada de células e diminuição das lacunas entre os corneócitos, portanto, melhorando a permeação da droga e fornecendo um tratamento tópico eficaz (WAGHULE et al., 2020). 
Por fim, alguns artigos da amostra final mencionaram que seus SLTF apresentaram redução dos efeitos adversos (AGRAWAL et al., 2020; KIM et al., 2018) e baixa toxicidade (SAPINO et al., 2017). Estes são aspectos relevantes pois beneficiam os pacientes acometidos por psoríase ao reduzir a carga do tratamento. Além disso, considerando que a dose terapêutica pode ser reduzida com o desenvolvimento de métodos avançados de SLFT, observa-se que esta estratégia de tratamento oferece melhor viabilidade e consistência para o paciente (KUMAR et al., 2020).

Somado às características supracitadas acerca dos SLTF, outras propriedades destes sistemas, como evitar o metabolismo de primeira passagem e possibilidade de liberação de medicamentos controlada e à longo termo, são as principais vantagens da incorporação desta técnica de administração parenteral no plano terapêutico dos pacientes (QI, 2020; NEUPANE et al., 2020).

\section{CONCLUSÕES}

Após a análise dos artigos da amostra final, identificou-se o uso de nove sistemas de liberação de fármacos transdérmicos utilizados no tratamento de psoríase. Os artigos destacaram a eficácia destes sistemas na entrega de drogas anti-inflamatórias através da pele. Além disso, esta técnica de administração parenteral de medicamentos oferece menos riscos ao paciente, menos efeitos colaterais, redução de dose e, consequentemente, carga do tratamento.

Com esta pesquisa identificou-se ainda uma escassez de publicações sobre sistemas de liberação de fármacos transdérmicos no tratamento de psoríase, portanto, sugere-se o desenvolvimento de mais pesquisas sobre a temática.

\section{REFERÊNCIAS}

AGRAWAL, Yogeeta O. et al. Methotrexate-Loaded Nanostructured Lipid Carrier Alleviates Imiquimod-Induced Psoriasis by Moderating Inflammation: Formulation Optimization, Characterization, In-Vitro and In-Vivo Studies. International Journal of Nanomedicine, v. 15, p. 4763, 2020.

AGOSTINI, Tatiane; SILVA, D. Ácido hialurônico: princípio ativo de produtos cosméticos. Santa Catarina, 2010. Disponível http://siaibib01.univali.br/pdf/Tatiane\%20Agostini.pdf. Acesso em: 05 de outubro 2020

BABY, André Rolim et al. Estabilidade e estudo de penetração cutânea in vitro da rutina veiculada em uma emulsão cosmética através de um modelo de biomembrana alternativo. Revista Brasileira de Ciências Farmacêuticas, v. 44, n. 2, p. 233-248, 2008. 
BARDIN, Laurence. Análise de conteúdo. São Paulo: Edições 70, 2011, p. 229.

BOAKYE, Cedar HA et al. Novel amphiphilic lipid augments the co-delivery of erlotinib and IL36 siRNA into the skin for psoriasis treatment. Journal of Controlled Release, v. 246, p. 120$132,2017$.

BOEHNCKE, Wolf-Henning; BREMBILLA, Nicolo Costantino. Unmet needs in the field of psoriasis: pathogenesis and treatment. Clinical Reviews in Allergy \& Immunology, v. 55, n. 3, p. 295-311, 2018.

DEPIERI, Lívia Vieira et al. RNAi mediated IL-6 in vitro knockdown in psoriasis skin model with topical siRNA delivery system based on liquid crystalline phase. European Journal of Pharmaceutics and Biopharmaceutics, v. 105, p. 50-58, 2016.

DUNCAN, J. I. et al. Immunomodulation of psoriasis with a topical cyclosporin A formulation. Acta dermato-venereologica, v. 73, n. 2, p. 84-87, 1993.

GOMES, R. K.; GABRIEL M. Cosmetologia: Descomplicando os Princípios Ativos. $2^{\mathrm{a}}$ ed. São Paulo: Livraria Médica Paulista Editora. 2006.

GORZELANNY, Christian et al. Skin Barriers in Dermal Drug Delivery: Which Barriers Have to Be Overcome and How Can We Measure Them?. Pharmaceutics, v. 12, n. 7, p. 684, 2020.

JUNQUEIRA, L. C.; CARNEIRO, J.; ABRAHAMSOHN, P. Histologia básica: texto e atlas. 13. ed. Rio de Janeiro: Guanabara Koogan, 2017

KIM, Jin Yong et al. Nanoparticle-assisted transcutaneous delivery of a signal transducer and activator of transcription 3-inhibiting peptide Ameliorates psoriasis-like skin inflammation. ACS nano, v. 12, n. 7, p. 6904-6916, 2018.

KUMAR, Bhumika et al. Novel Topical Drug Delivery Approaches for the Management of Psoriasis. International Journal of Pharmaceutical Sciences and Nanotechnology, v. 13, n. 5, p. 5062-5068, 2020.

METHLEY, Abigail M. et al. PICO, PICOS and SPIDER: a comparison study of specificity and sensitivity in three search tools for qualitative systematic reviews. BMC health services research, v. 14, n. 1, p. 579, 2014.

NEUPANE, Rabin et al. Alternatives to biological skin in permeation studies: Current trends and possibilities. Pharmaceutics, v. 12, n. 2, p. 152, 2020.

PARISI, Rosa et al. National, regional, and worldwide epidemiology of psoriasis: systematic analysis and modelling study. bmj, v. 369, 2020.

PAWLINA, W.; ROSS, M. H. Histology: a text and atlas: with correlated cell and molecular biology. Lippincott Williams \& Wilkins, 2018.

QI, Qin M. et al. Comparison of Ionic Liquids and Chemical Permeation Enhancers for Transdermal Drug Delivery. Advanced Functional Materials, p. 2004257, 2020. 
RANADE, Vasant V. Drug delivery systems. 6. Transdermal drug delivery. The Journal of Clinical Pharmacology, v. 31, n. 5, p. 401-418, 1991.

RAPALLI, Vamshi Krishna et al. Psoriasis: pathological mechanisms, current pharmacological therapies, and emerging drug delivery systems. Drug Discovery Today, 2020.

SALA, M.; ELAISSARI, A.; FESSI, H. Advances in psoriasis physiopathology and treatments: Up to date of mechanistic insights and perspectives of novel therapies based on innovative skin drug delivery systems (ISDDS). Journal of Controlled Release, v. 239, p. 182-202, 2016.

SAPINO, Simona et al. Mesoporous silica nanoparticles as a promising skin delivery system for methotrexate. International journal of pharmaceutics, v. 530, n. 1-2, p. 239-248, 2017.

SEBASTIA-SAEZ, Daniel et al. New trends in mechanistic transdermal drug delivery modelling: Towards an accurate geometric description of the skin microstructure. Computers \& Chemical Engineering, p. 106976, 2020.

SHAH, Punit P. et al. Skin permeating nanogel for the cutaneous co-delivery of two antiinflammatory drugs. Biomaterials, v. 33, n. 5, p. 1607-1617, 2012.

SOCIEDADE BRASILEIRA DE DERMATOLOGIA (SBD) REALIZA PESQUISA INÉDITA NA AMÉRICA DO SUL. Sociedade Brasileira de Dermatologia, 2020. Disponível em: <http://www.sbd.org.br/psoriasetemtratamento/noticias/informe-se/sociedade-brasileirade-dermatologia-sbd-realiza-pesquisa-inedita-na-america-do-sul/>. Acesso em: 31, Outubro, 2020.

SOMAGONI, Jaganmohan et al. Nanomiemgel-a novel drug delivery system for topical application-in vitro and in vivo evaluation. PLoS One, v. 9, n. 12, p. e115952, 2014.

SOUZA, M. T.; SILVA, M. D.; CARVALHO, R. Integrative review: what is it? How to do it?. Einstein.(São Paulo)[Internet]. 2010 Mar [citado 31 Outubro 2020]; 8 (1): 102-6.

VELASCO, Maria Valéria Robles et al. Rejuvenescimento da pele por peeling químico: enfoque no peeling de fenol. Anais brasileiros de dermatologia, v. 79, n. 1, p. 91-99, 2004.

WADHWA, Sheetu et al. Liposomal fusidic acid as a potential delivery system: a new paradigm in the treatment of chronic plaque psoriasis. Drug delivery, v. 23, n. 4, p. 1204-1213, 2016.

WAGHULE, T., SANKAR, S., RAPALLI, V. K., GORANTLA, S., DUBEY, S. CHELLAPPAN, D. K., SINGHVI, G. (2020). Emerging Role of Nanocarriers based Topical delivery of Anti-Fungal Agents in Combating Growing Fungal Infections. Dermatologic Therapy.

WHITTEMORE, R. and KNAFL, K. (2005) The integrative review: updated methodology. Journal of Advanced Nursing 52: 546-553. 